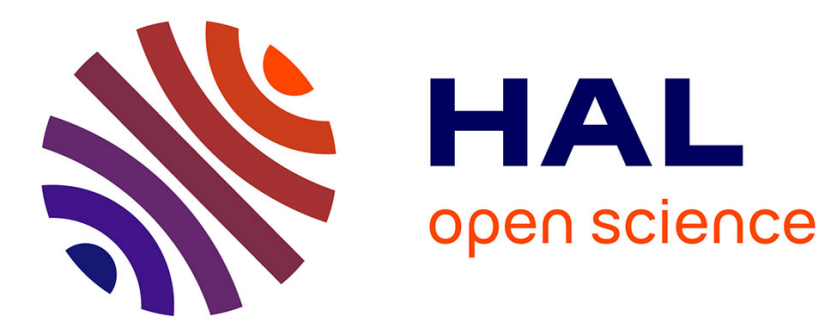

\title{
Pratiques langagières et registres discursifs
}

Emmanuelle Cambon, Isabelle Léglise

\section{To cite this version:}

Emmanuelle Cambon, Isabelle Léglise. Pratiques langagières et registres discursifs: Interrogation de deux cadres en sociologie du langage. Langage et Société, 2008, 124, pp.15-38. halshs-00292261

\section{HAL Id: halshs-00292261 https://shs.hal.science/halshs-00292261}

Submitted on 30 Jun 2008

HAL is a multi-disciplinary open access archive for the deposit and dissemination of scientific research documents, whether they are published or not. The documents may come from teaching and research institutions in France or abroad, or from public or private research centers.
L'archive ouverte pluridisciplinaire HAL, est destinée au dépôt et à la diffusion de documents scientifiques de niveau recherche, publiés ou non, émanant des établissements d'enseignement et de recherche français ou étrangers, des laboratoires publics ou privés. 
Cambon, Emmanuelle \& Léglise, Isabelle (2008). Pratiques langagières et registres discursifs : Interrogation de deux cadres en sociologie du langage. Langage et Société 124, 15-38

\title{
Pratiques langagières et registres discursifs Interrogation de deux cadres en sociologie du langage
}

\author{
Emmanuelle Cambon \\ Université Paris III, emcambon@aliceadsl.fr \\ Isabelle Léglise \\ CNRS, UMR 8133 CELIA, leglise@vjf.cnrs.fr
}

Ce texte cherche à mettre en évidence les liens, les incompatibilités, les articulations entre deux cadres théoriques dont la visée explicite est une sociologie du langage, celle, initialement exprimée par Josiane Boutet, Pierre Fiala et Jenny Simonin-Grumbach en 1976, qui proposent d'envisager des pratiques langagières constitutives d'une formation langagière, et celle, présentée par Pierre Achard (1995a), qui propose de parler de registres discursifs constitutifs d'une formation discursive. Partageant un refus de la théorie du reflet et considérant le langage ou le discours comme un acte dans le monde davantage que comme une représentation de celui-ci, partageant également une réflexion sur les rapports entre langage et fonctionnement social, ces deux cadres n'entretiennent pourtant pas de rapports explicites l'un avec l'autre. Si P. Achard, dans différents écrits, fait usage des notions mises en place par l'autre cadre (en particulier la notion de formation langagière) en y faisant référence, il semble que ce soit néanmoins de façon problématique. J. Boutet et P. Fiala, pour leur part, n'utilisent pas les notions mises en place par P. Achard et - à notre connaissance - n'y renvoient pas. Au-delà des raisons qui pourraient expliquer cet état de fait, nous voulons interroger ces différentes notions, en particulier celles de pratique langagière et de registre discursif, dans les rapports qu'elles peuvent entretenir. Il nous semble en effet important de dégager si ce n'est une théorie unifiée, du moins des éléments d'unification pour une sociologie du langage.

L'idée d'un rapprochement entre ces deux cadres vient des deux couples de notions que chacun développe. On est en effet en présence de pratiques langagières, d'une part, et de registres discursifs, d'autre part, qui sont reliés à une formation, respectivement, langagière / discursive. Notre question, à partir de là, est de savoir si l'on a affaire à deux objets empiriques distincts, pratiques et registres, ou à deux points de vue différents sur un même objet empirique, le langage envisagé dans son rapport aux fonctionnements sociaux. Dans les deux cas, on a affaire à deux objets empiriques différents, mais s'il s'agit de deux points de vue sur le langage et le social, on est conduit à s'interroger sur la sociologie du langage susceptible de se dégager d'une articulation entre les deux cadres et les deux couples de notions.

Dans le cadre de cet article, nous ne pourrons qu'avancer quelques pistes dans ce sens. En revanche, nous nous interrogerons davantage sur la compatibilité ou l'opposition des deux sociologies du langage à l'horizon de ces deux cadres. Dans un premier temps, ces différentes notions seront présentées à travers des définitions et des exemples. Il nous paraît en effet important de revenir aux textes tant le métalangage employé est, actuellement, polysémique. "Pratiques langagières » a eu un succès important mais a perdu beaucoup du sens proposé par ses concepteurs; il est souvent employé comme synonyme à "pratiques linguistiques », «interactions verbales » ou «prises de parole». «Registre » et « genre » pour leur part sont utilisés aussi bien en sociolinguistique, en analyse textuelle qu'en analyse de discours, avec des acceptions très différentes selon les traditions. Nous estimons que seul le dialogue avec les textes, en contexte, peut permettre de rendre audible les concepts manipulés. 
Dans un deuxième temps, nous présenterons les rapports que ces pratiques, registres et genres entretiennent aux formations (langagière ou discursive). Après avoir noté quelques-unes de leurs ressemblances et divergences, nous avancerons quelques propositions d'articulation entre ces deux cadres. Nous verrons que la question du discours, qui pourrait n'apparaître que terminologique, devient alors cruciale.

\section{UN POSTULAT COMMUN}

Les deux cadres cités considèrent l'activité langagière comme une pratique sociale à part entière ; néanmoins, celle-ci est transversale et n'est pas passible d'un traitement «régional» dans une sociologie, au même titre, par exemple, que l'analyse des pratiques maritales, vestimentaires ou de travail. Ainsi lit-on chez J. Boutet (1985:6) :

«... nous défendons l'idée que la pratique de langage ou pratique langagière est à considérer comme une pratique sociale et à analyser comme telle; c'est-à-dire que les mots, les discours, ne sont pas seulement les représentants de nos actes et de nos pensées, ils ne sont pas seulement là pour transmettre de l'information ou des idées ou des ordres. Ils ne se contentent pas de refléter le social, ils en sont partie prenante et ils agissent sur le social, y produisant des effets spécifiques que l'analyste doit repérer [...]. Tout acte, qu'il soit technique ou pas est conçu, représenté par des acteurs sociaux, c'est-à-dire travaillé dans le matériau sémiotique qu'est le langage. »

chez Marianne Ebel et Pierre Fiala (1983 : 156) :

«Dans leur diversité, ces pratiques [langagières] ne sauraient toutefois être seulement envisagées comme "reflet" des rapports économiques ou "traces" de rapports de force sociaux, idéologiques. Elles interviennent en tant que telles dans les domaines de la vie sociale et avec des effets matériels qui sont liés directement à leur exercice. [...] les pratiques langagières ont, dans leur spécificité, des effets sociaux immédiats liés à leurs formes mêmes et aux conditions dans lesquelles elles s'exercent. »

ou chez P. Achard (1995a : 84) :

«... le discours n'est pas la "face langagière" de l'acte, mais l'interdépendance de la face langagière et de la face pratique. La face langagière n'est pas un simple "reflet" de la face pratique. Si un acte discursif peut parfois comporter une "injonction à faire" (perlocutoire), l'interpellation des acteurs-locuteurs à des places catégorisées est un acte performatif. »

Chacun insiste donc sur les effets dans le monde qu'a le langage. Ce postulat, la dimension d'acte du langage, nous semble toutefois un carrefour dans la mesure où, à partir de cette affirmation, deux directions différentes sont prises, qui soutiennent les traitements et les visées dans les deux sociologies du langage proposées.

\section{DEFINITIONS}

Les deux cadres conceptuels mettent en lien deux «niveaux » qu'on prendra comme niveau d'analyse des données : d'une part un certain rapport entre des pratiques sociales et des formes linguistiques, les pratiques langagières ou les registres discursifs, d'autre part un rapport entre ces pratiques et un arrière-plan qui les organise et dont elles sont constitutives, la formation langagière ou la formation discursive. Il y a un rapport de constitution et d'influence (peut-être de structuration) entre ces deux niveaux.

\subsection{Formation langagière et pratiques langagières}

La première définition de formation et pratique langagières (1976) trouve place dans une lecture critique de deux ouvrages traitant du rapport entre langue, discours et organisation sociale, celui de J.-B. Marcellesi et B. Gardin (1974) et celui de R. Robin (1973) : 
«Nous voulons tenter d'appréhender un ensemble de pratiques langagières, hiérarchisées socialement, spécifiques d'une formation sociale donnée ; ensemble dans lequel tout locuteur est pris sans même en être conscient. Nous avançons l'idée d'une formation langagière, entendue comme un ensemble réglé de pratiques langagières, qui organise celles-ci selon un rapport de force en pratiques dominantes et pratiques dominées. En ce sens, il s'agit de décrire les rapports de force qui structurent les pratiques langagières au sein d'une formation langagière. De ce fait, nous posons qu'il existe des rapports de pouvoir entre les pratiques langagières, et non pas seulement, comme dans la perspective de Robin ou de Marcellesi et Gardin, que le langagier porte trace, reflète des rapports de pouvoir existant ailleurs. » (Boutet et alii $1976: 83$ )

Cette définition vient après la présentation de phénomènes langagiers observés au cours d'une enquête ${ }^{1}$ sur une chaîne de montage en usine ; l'absence d'échange ou des échanges très brefs entre les travailleurs, y compris pendant les pauses, conduit les auteurs à considérer ce «débit » comme une pratique langagière particulière qu'une transcription des échanges n'aurait pas pu mettre en valeur. Ainsi il s'agit d'insister sur un point de vue désormais classique qui envisage la situation pratique au sein de laquelle se déroulent les échanges langagiers afin de les caractériser. On note l'insistance des auteurs sur les rapports de pouvoir qui se déroulent entre les pratiques langagières, ce qui leur confère un statut «d'instances agissantes ».

\section{Formation langagière}

Une formation langagière se définit de deux points de vue : elle est un «ensemble partiellement réglé de pratiques langagières différenciées » et elle structure les rapports entre les pratiques langagières en termes de dominance et de rapports de forces. La formation langagière est, à son tour, partie intégrante d'une formation sociale, ce qui est une autre façon de refuser la théorie du reflet et de l'extériorité du langage par rapport au «social» et de poser le langagier comme «facteur constitutif d'une société déterminée et, comme tel, dépendant de l'organisation de la formation sociale » (Boutet et alii 1976 : 80). L'effet des pratiques langagières, en tant que pratiques, qu'actes, se manifeste dans les rapports sociaux :

«En tant que pratique multiple, le langage est partie intégrante des formations sociales non seulement dans la production des discours, mais aussi dans leur circulation, non seulement comme produit des rapports qui les structurent mais aussi comme produisant des transformations dans ces rapports.

La notion de formation langagière, entendue comme un ensemble partiellement réglé de pratiques langagières différenciées, prend ici son sens. Élément constitutif d'une formation sociale, une formation langagière organise ces pratiques diverses selon des rapports de forces en pratiques dominantes et pratiques dominées. » (Ebel et Fiala 1976: 155, nous soulignons).

On est ainsi en présence d'un rapport dynamique entre pratiques et formation puisque celle-ci « organise » les pratiques et que ces dernières, en retour, «transforment» les rapports sociaux, tributaires de la formation langagière et de la formation sociale. On trouve le même type de relation dynamique dans le second cadre conceptuel, entre formation discursive et registre discursif.

La difficulté à saisir ce qu'est une formation langagière vient probablement de son caractère conceptuel, à la limite de l'insaisissable ${ }^{2}$. L'usage qu'en font certains auteurs

\footnotetext{
${ }^{1}$ I. Granstedt, communication au groupe de sociologie du travail, Dpt de sociologie de Paris VII (hiver 1975).

${ }^{2}$ Là encore, on retrouve cette caractéristique pour la notion de formation discursive.
} 
peut cependant permettre d'en dégager des propriétés. Ainsi, J. Boutet propose-t-elle de parler de

" "formation langagière du travail" construite sur la relégation des pratiques langagières issues des salariés et sur la domination de celles issues de l'organisation et de l'encadrement. Ce rapport de force rend compte du fait que les salariés disent tous avoir de grandes difficultés à parler de leur travail car peu de discours existent, circulent, qui constitueraient un "flux dialogique" au sein duquel les salariés pourraient se situer pour reprendre, paraphraser, argumenter. » (Boutet $2002: 273$, nous soulignons).

L'article de 1976 fournit selon nous un autre exemple de formation langagière :

«... décrire, par exemple au sein de la formation sociale française actuelle, la place occupée par l'ensemble des pratiques langagières qu'on appelle "le français" ». (Boutet et alii 1976 : 81 , nous soulignons)

puisque la formation a été définie comme ensemble de pratiques; autrement dit, ce qu'on appelle «le français» serait une formation langagière, ce qui permet de considérer la langue (en tant qu'institution appareillée, par l'école en particulier) dans son aspect non homogène et comme sujette à variations du fait de sa prise dans le fonctionnement social.

Mais une formation langagière semble beaucoup mieux se définir en fonction des liens qu'elle entretient avec les pratiques qui la constituent et sur lesquelles elle pèse. L'analyse, menée par M. Ebel et P. Fiala (1983), de la xénophobie en Suisse, lors des campagnes de votation en 1970, 1974 et 1977 concernant la restriction de l'immigration dans ce pays met à contribution ces deux notions en les articulant; le contrôle de la production et de la circulation des discours en constitue le point nodal. On y voit également que des institutions, comme les partis politiques, le vote, les médias par exemple, cadrent la formation langagière qui à son tour règle les rapports entre pratiques langagières :

«On pourrait utiliser une image pour caractériser le réglage des pratiques dans les campagnes de votations : celle d'un entonnoir constituant un goulot d'étranglement dans lesquels les discours se resserrent pour ne constituer finalement que les trois énoncés normés et homogènes : OUI, NON, JE M'ABSTIENS.

Sans pouvoir décrire exhaustivement le fonctionnement d'une formation langagière à un moment de son histoire, il est possible de repérer, lors des campagnes, certains des mécanismes qui réduisent les produits des pratiques hétérogènes. Nous avons noté déjà comment l'institution de vote règle la circulation des discours par les limites temporelles qu'elle impose à la campagne mais aussi aux locuteurs contraints d'exprimer leur position par un bulletin de vote; les partis politiques, toutes les organisations représentatives exprimant des positions à travers des discours normés, constituent un cadre qui donne sa forme dominante à la formation langagière. Les médias sont un autre élément fondamental de ce réglage institutionnel de pratiques langagières.

[...] Ce type de contrôle qui règle la circulation même des discours contribue, comme l'ensemble des institutions, à imposer la forme et le contenu du débat et à présenter les votations comme conclusions naturelles de ce débat.» (Ebel-Fiala 1983: 172, nous soulignons)

Autrement dit, c'est dans ce qui apparaît comme un extérieur au langage, et qu'on caractérisera comme des institutions, que se règle, au final, le fonctionnement des pratiques langagières différenciées, la formation langagière jouant sur l'organisation des rapports entre ces pratiques. 


\section{Pratiques langagières}

Les pratiques langagières, pour leur part, sont davantage décrites. On peut rappeler la définition qu'en donne J. Boutet (2002: 459) :

«D'un point de vue empirique, "pratique langagière" renvoie aux notions de "production verbale", d'"énonciation", de "parole", voire de "performance", mais il s'en distingue d'un point de vue théorique par l'accent mis sur la notion de "pratique" : le langage fait partie de l'ensemble des pratiques sociales, que ce soit des pratiques de production, de transformation ou de reproduction. Parler de "pratique", c'est donc insister sur la dimension praxéologique de cette activité.

Comme toute pratique sociale, les pratiques langagières sont déterminées et contraintes par le social, et en même temps, elles y produisent des effets, elles contribuent à le transformer. Dans cette perspective, le langage n'est pas seulement un reflet des structures sociales mais il en est un composant à part entière. [...] Parler n'est pas seulement une activité représentationnelle, c'est aussi un acte par lequel on modifie l'ordre des choses, on fait bouger les relations sociales. »

Outre le postulat de départ (la dimension d'acte du langage), on trouve ici une indication sur ce qu'est empiriquement une pratique langagière : une donnée langagière observable en tant qu'elle a des effets sociaux. M. Ebel et P. Fiala (1983) ${ }^{3}$ travaillent la notion et la relient à la constitution d'une sociologie du langage. La dimension d' «acte social » ne semble cependant pas l'objet premier que permet de décrire la notion de pratique langagière dans son caractère opératoire ; pour les deux auteurs, elle permet de

«... décrire les processus de production du sens comme phénomènes discursifs produits à la fois par des rapports de forces sociaux dans des situations déterminées et par des facteurs (grammaticaux, rhétoriques) relevant de la mise en discours mais aussi des conditions de mise en circulation de ces discours. » (Ebel-Fiala 1983 : 156, nous soulignons)

M. Ebel et P. Fiala insistent sur le fait que «la réalité du signe est foncièrement conflictuelle » et que le signe est « un objet idéologique, socialement pluri-accentué, historique, et comme tel, lieu de la lutte des classes » et enjeu de cette dernière. Ils cherchent à montrer que « la classe dominante, à travers l'ensemble des manifestations de langage, tend à effacer la pluralité d'accents du signe et à le rendre mono-accentuel, étouffant ainsi " "la lutte des indices de valeur" dont le signe est l'objet » (ibid. 150), et ce sur les formules de «Ueberfremdung » ${ }^{4}$ et «xénophobie» lors des campagnes de votation des années 70 en Suisse:

«Envisager le langage comme agent et enjeu social, c'est montrer comment les pratiques langagières hétérogènes s'organisent dans diverses formes d'interactions verbales (débats, conversations, récits, polémiques) autour de référents construits et transformés dans les discours ainsi produits; ces pratiques sont portées par les agents sociaux, qui dans l'exercice du langage se constituent en sujets collectifs ou individuels; elles sont organisées selon des rapports de forces qui s'établissent de façons diverses : dans la prise de parole, dans la mise en jeu des règles qui norment les différents emplois de la langue, dans la construction et l'imposition des significations dans les discours, dans la mise en circulation des discours et dans les effets produits par cette mise en circulation.

Décrire les pratiques langagières en tant qu'elles produisent des significations et les transforment, décrire ces processus en tant qu'ils sont marqués par des rapports de domination, d'exclusion, de renforcement, les analyser comme pratiques sociales signifiantes, nous semble être un objectif important d'une sociologie du langage.» (ibid. 156).

\footnotetext{
${ }^{3}$ Cette notion est utilisée dans d'autres travaux, en particulier dans les travaux d'Elisabeth Bautier (1995) sur l'école.

${ }^{4}$ ou sentiment d'« envahissement par des étrangers ».
} 
Il s'agit ainsi de saisir, du point de vue langagier, la matérialité de la circulation des significations des deux formules ${ }^{5}$ Ueberfremdung et xénophobie en tant qu'elles sont parties prenantes de l'opinion publique :

«... ces deux termes ont, durant cette période, condensé en eux une masse considérable de discours, auxquels ils servaient d'équivalents sémantiques. Renoncer à l'un ou l'autre c'était mettre en circulation des significations multiples, contradictoires, renvoyant à l'existence de séries d'énoncés paraphrastiques bien attestés, par lesquelles les deux termes se trouvaient définis. » (ibid. 173) ${ }^{6}$.

Le dialogisme attaché à ces deux formules est ainsi le point central analysé par M. Ebel et P. Fiala, en tant qu'il s'organise dans le matériau langagier. Après avoir observé le contrôle exercé sur les lettres de lecteurs par le journal, les deux auteurs analysent le réseau argumentatif entre une dizaine de lettres de lecteurs; celles-ci constituent en effet, publiées ou non, un dialogue puisqu'elles sont des réponses les unes aux autres. L'accusation de xénophobie apparaît comme référent social ${ }^{7}$ dominant, contraignant tout locuteur à se situer par rapport à elle, et son rejet est ce qui fait l'homogénéité des lettres, tandis que les diverses formes linguistiques observées attestent de leur hétérogénéité. Néanmoins, le caractère homogène de cette pratique langagière tient aussi à un facteur extérieur, la situation et ses caractéristiques institutionnelles. Notant que les lettres de lecteurs ne présentent pas un caractère unifié d'un point de vue linguistique, typographique ou en ce qui concerne les supports, les deux auteurs voient dans la situation un principe d'unification de la pratique langagière :

«La disparité de ces matériaux pourrait faire conclure à l'impossibilité d'une analyse systématique. En deçà de ce caractère hétéroclite, ces lettres relèvent pourtant d'une pratique langagière spécifique déterminée par une situation institutionnalisée : dans le cours d'une campagne politique relayée par la presse, des lecteurs, pour de raisons explicites ou non, et avec des moyens langagiers propres à chacun, ont pris part à un débat lancé par leur journal. Là est le point d'unification de cette pratique. Parce qu'ils se sentaient directement concernés par une campagne politique qui se développait jours après jours sous leurs yeux, et qu'ils jugeaient décisifs les enjeux du débat, ces lecteurs ont pris la plume. » (ibid. 182, nous soulignons).

Ce n'est donc qu'après avoir défini une unité de la pratique langagière par des caractéristiques non langagières qu'il est possible d'observer la récurrence de certaines formes linguistiques et de les analyser de façon systématique.

\subsection{Formation discursive et registre discursif}

P. Achard a adopté une perspective tout à la fois cumulative et restrictive par rapport à l'Ecole française d'Analyse de discours, avec l'objectif de refonder les principes de l'analyse sociologique à l'aune de la description des phénomènes langagiers en tant que partie prenante de la réalité sociale. Ainsi, son utilisation de la notion de formation

\footnotetext{
5 «Formule » est entendu au sens de Jean-Pierre Faye (2004) : citation.

${ }^{6}$ Des énoncés paraphrastiques sont donnés en exemple, tirés de brochures «anti-xénophobes »: «les étrangers ne connaissent pas notre langue et ne font aucun effort pour l'apprendre / ils font du bruit / ils nous prennent notre argent...».

7 « Nous entendons par là que dans les années 60-80 tout locuteur, individuel ou collectif, savait ou prétendait savoir ce que "signifiaient" ces formules. Cela n'implique pas, bien sûr, que tous leur donnaient la même signification, mais seulement qu'elles appartenaient l'une et l'autre à la formation langagière propre à la Suisse romande, comme en font partie d'autres formules telles que patrie, neutralité, indépendance nationale, communisme, liberté, étranger (à la différence de formules qui existent dans d'autres pays ou à d'autres époques, mais n'ont pas en Suisse actuellement de référent social ; par exemple, révolution nationale, impérialisme, autogestion ouvrière, nationalisation, etc.) », Ebel-Fiala 1983 : 174.
} 
discursive est-elle en même temps rattachée à la réflexion qui a été menée autour de cette notion et retravaillée, avec des hypothèses fortes, afin de poser les jalons d'une théorie du fonctionnement social en appui sur les propriétés générales du discours.

\section{La notion de formation discursive}

La notion de formation discursive est essentiellement rattachée à deux auteurs, Michel Foucault, qui la propose dans L'Archéologie du savoir (1969), et Michel Pêcheux, dans Analyse automatique du discours (1969). Pour datée qu'elle puisse paraître et même si elle a subi nombre de remaniements qui, au final, rendent difficile une définition consensuelle, elle est néanmoins centrale dans la sociologie du langage proposée par Achard. En quoi cette notion demeure-t-elle un concept pertinent et opératoire ? C'est qu'il s'agit de maintenir avec cette notion ce qui ressort de la réflexion de Foucault : l'arrière-plan historique sur lequel on voit des dires catégorisés comme relevant d'un même ensemble est un arrière-plan discursif. C'est via la référence à Foucault que, par exemple, Achard porte une critique à la sociologie du langage de Bourdieu (1982) :

«Le texte de Michel Foucault (1971) cristallise un certain nombre d'idées diffuses qui soustendent, me semble-t-il, toute la pratique de l'analyse de discours dans l'acception 'française' de cette notion. On peut en résumer l'essentiel en disant que la littéralité du texte (son mot à mot, et non pas un 'sens littéral' qu'on ne suppose justement pas) ne suffit pas à dégager l'organisation discursive, mais qu'il faut de plus postuler une organisation discursive d'ensemble : un texte donné ne constitue jamais seul un discours, il prend sens par l'hypothèse qu'il fait bien partie de telle série, distincte de telle autre, et qu'il y fait événement, et que telle autre série d'événements en est distincte. De plus, dire qu'il y a 'événement', cela implique que le discours effectif n'est pas prédéterminé par ses 'conditions de production (Henry et Moscovici, 1968) ; tout au plus est-il contraint par celles-ci (cf. les 'interdits' Foucault 1971), menacé d'exclusion s'il déborde du cadre permis, et interprété quant au contenu, à la 'discipline', à la validité de son énonciation. La réflexion de Michel Foucault, par son existence, interdit de faire de l'analyse de discours une démarche causale descendante, du type : sens + conditions sociales $\rightarrow$ discours.

Il affirme l'hypothèse, qu'on ne peut que suivre, que le discours (événement) est une forme (relativement arbitraire) dont le sens est construit par des procédures sociales qui lui assignent un statut (on rejoint ici l'analyse de P. Bourdieu), et que ces procédures sociales sont elles-mêmes d'ordre discursif (ce que le texte de Bourdieu néglige), donc événementielles » (Achard $1986: 15-16$, nous soulignons).

La formation discursive, en tant que « structure implicationnelle d'affectation d'effet de sens aux registres » peut être reliée à ces «procédures sociales » qui assignent un statut à un texte, c'est-à-dire qui le situent dans une série, un registre discursif.

\section{Le dialogisme et la question de la frontière}

Il convient de rappeler que la prise en compte du dialogisme ou de l'hétérogénéité constitutive de tout discours, est ce qui, dans le courant de l'AD en France dans les années 80, a mis en question la notion de formation discursive de par la clôture qu'elle imposait autour d'un ensemble de discours, tandis que le dialogisme mettait en évidence une absence de clôture et ouvrait la voix à l'importance de l'interdiscours dans l'intradiscours ( $c f$. Marandin et Courtine 1981). On peut souligner l'acception double et dont les deux membres sont articulables - de la notion de dialogisme, ce qui nous permettra de préciser en quoi, chez Achard, la notion de FD est maintenue :

d'une part le dialogisme rend compte du fait que le «mot vit sa vie de mot» (Bakhtine) et qu'il est pluriaccentué, polyphonique, chambre d'écho des usages qui en ont été faits ; 
- d'autre part le dialogisme renvoie aussi, dans les écrits de Bakhtine-Volochinov, au fait que les signes linguistiques sont des signes idéologiques, du fait même de cette pluriaccentuation ; autrement dit, le dialogisme constituant de tout signe linguistique en usage n'empêche pas (ou rarement) que le signe soit rendu mono-accentuel de par les rapports de domination idéologique en présence.

Il nous semble donc important de souligner que la notion de formation discursive chez Achard, met 'hors champ opératoire' la question de la clôture de la formation discursive, en prenant justement en compte le fait qu'on ne peut pas mettre en évidence de frontière, $d u$ fait $d u$ dialogisme entendu ici au sens de pluriaccentuation, même si l'on peut mettre en évidence des "fêlures" ou ruptures stables entre registres. Il traite de la FD en termes de centres attracteurs et c'est ce qui permet de penser la deuxième acception de dialogisme, à savoir celle d'une accentuation idéologique dominante des signes.

Il ne s'agit certainement pas de nier l'événement que peut être un énoncé, mais seulement de se donner les moyens de faire de la sociologie en prenant au sérieux la place du langage ou plutôt celle du discours. Il s'agit de prendre en considération le fait que ces centres écrasent la multiplicité et l' "essaim " ${ }^{8}$ des significations qui se lève dès lors que l'on énonce. Ce n'est qu'à cette condition qu'on peut faire de la sociologie, dont l'un des objets est, selon Achard, la description de l'institution :

«L'institution, objet de la sociologie, est la structure synchroniquement stable des types d'actes, avec les places qui leur sont associées. » (Achard 1995a : 83)

Et l'institution ne peut être que stable par définition. Parler de centre plutôt que d'ensemble c'est permettre que demeure ouverte la possibilité des interprétations et des attestations événementielles, faisant «bougé » (Pêcheux 1990: 323) et, partant, déstabilisant l'institution en question. Il faut, pensons-nous, insister sur le caractère synchronique de sa conception de la formation discursive, ce qui est un changement de perspective par rapport aux définitions initiales, de Foucault mais aussi de Pêcheux. Elle permet certes de renvoyer à l'histoire et à la relativité de la structuration des actes ; elle permet également de retrouver la ou les filiation(s) d'un registre discursif (c'est-à-dire d'une pratique sociale), c'est-à-dire ce qui historiquement la fait stable, acceptable, évidente et donc relevant de l'idéologique. Mais, conçue comme les rapports synchroniques entre des registres discursifs, elle permet de décrire, c'est le but idéal, la structuration d'une «société ».

Quant à la «totalité » de la FD, elle n'apparaît pas objectivable, puisqu'on ne peut mettre de frontière en évidence, mais elle est pourtant nécessaire, en tant qu'horizon de clôture, afin de pouvoir saisir des stabilisations fonctionnelles que nous assimilons aux registres discursifs.

\footnotetext{
${ }^{8} C f$. Authier 1995 : 718, d'après Lacan 1966.
} 


\section{Registres discursifs ${ }^{9}$}

La notion de formation discursive est centrale pour l'étayage théorique de la sociologie achardienne : une formation discursive «est la structuration de l'espace social par différenciation des discours » (Achard 1995 : 84). Elle constitue une assise pour, dans l'analyse, effectuer une «cartographie discursive», "procédé de description systématique du social comme formation discursive » (Rapport SLADE 1996 : 150). On comprend, à partir de la citation, qu'il y ait nécessité à déniveler la formation discursive et à proposer un concept qui permette d'observer le résultat de cette différenciation, processus qui produit des discours, entendus au sens d'unités, comme ce que l'on a coutume d'appeler «le discours de la médecine», «le discours politique », «le discours de la publicité », les unités se soutenant d'un rattachement, objectivement sujet à caution, à des sources institutionnelles.

Les registres discursifs résultent donc de la différenciation, effectuée par la formation discursive, de ce qui apparaît, alors, comme des discours différents. Autrement dit, les registres discursifs sont le résultat d'un processus discursif tenant non tant à la «consistance » desdites « unités » qu'aux rapports qu'elles entretiennent entre elles.

S'ajoutent à cette différenciation, tenant à «l'ordre du discours » dont les formations discursives apparaissent comme agents (non exclusifs), les catégorisations des acteurs, c'est-à-dire leurs interprétations des énoncés : selon qu'ils les interprètent de telle ou telle façon, les attestés relèveront davantage de telle ou telle pratique sociale et, partant, de tel ou tel registre discursif. L'interprétation est, de ce fait, conçue ici comme une mise en série d'un énoncé avec d'autres qui lui sont voisins. On peut voir là un fondement objectif à la définition du registre comme «accumulation de textes dans un même voisinage ». Il faudrait alors savoir à quoi tient le fait qu'une interprétation n'est pas 'sans rivage' ${ }^{10}$; deux aspects sont à prendre en considération : le lest que fait peser l'ordre du discours et le fait que les signes linguistiques ont des valeurs stables qui font que, socialement, ils ne signifient pas n'importe quoi.

On retrouve, dans le premier aspect, la tendance classique à voir dans la notion de formation discursive ce qui leste les interprétations; le second aspect renvoie à ce que P. Achard appelle le genre discursif et qui concerne l'apparition des formes. Autrement dit, on serait là en présence d'une articulation entre contraintes sur l'interprétation des formes linguistiques et contraintes sur leur production.

\section{Registre et genre discursif}

Le genre chez Achard est toujours associé à un registre. C'est par là qu'on peut saisir la matérialité discursive d'une pratique sociale. Nous soulignons ici les précisions apportées quant au rapport non-arbitraire entre genre et registre. C'est probablement sur ce point en effet que les deux cadres en sociologie du langage divergent le plus fondamentalement.

Si l'on suit sa définition, on est conduit à postuler des invariants linguistiques :

«[les] registres fonctionnent sur la base de régularités relativement indépendantes de la mise en acte, les formes linguistiques: l'analyse des jeux de langage ne peut aboutir à une grammaire, elle la présuppose. Au registre pourra donc être associé un genre, c'est-à-dire une répartition inégale des formes. A la différence du point de vue variationniste (qui vise le système linguistique), cette répartition ne sera pas considérée comme arbitraire, mais comme

\footnotetext{
${ }^{9} C f$. également l'article de M. Sassier, ici même.

${ }^{10}$ Pour reprendre l'expression de Pêcheux.
} 
motivée par un rapport entre formes et fonctionnements sociaux. On verra cette motivation dans l'énonciation » (Achard 1995a : 84, nous soulignons).

S'il est clair que ce rapport renvoie à la nécessité de rapporter l'interprétation des formes au jeu de langage dans lequel elles sont attestées et interprétées, la production même des formes est ici en jeu et que, ces dernières se répétant, on doit voir une motivation à cette répétition (autrement dit, on pose qu'il y a des raisons à ce qu'une forme se répète). Aussi proposons-nous de dire que l'invariant des formes qui se répètent, là et non ailleurs, est socialement investi par le registre en question. C'est ce que nous comprenons de l'affirmation selon laquelle les contraintes institutionnelles pesant sur la répartition des rôles sociaux se répercutent sur l'usage des formes (Achard $1995 b: 8-9)$.

Considérer qu'il existe des registres discursifs n'est pas un artefact conceptuel, une façon commode d'entrer dans des données langagières avec des hypothèses sociologiques ou relevant d'autres sciences humaines; un quadrillage du flux qu'est le discours existe qui leste les interprétations, qui barre la route à l'entrée intempestive d'énoncés non «contrôlés ». La notion de registre, toute hypothèse qu'elle soit parce qu'elle demande analyse et, partant, méthode, est une façon d'en appréhender la teneur et de se donner les moyens d'en analyser la matérialité. Un registre est, objectivement, partie prenante du contrôle du discours.

\subsection{Synthèse}

Le tableau suivant propose une synthèse des différentes notions abordées.

\begin{tabular}{|c|c|}
\hline Formation langagière & Formation discursive \\
\hline $\begin{array}{l}\text { - Ensemble réglé de pratiques qui les organise selon des } \\
\text { rapports de force en pratiques dominantes et pratiques } \\
\text { dominées (Boutet et al. } 1976 \text { ) }\end{array}$ & $\begin{array}{l}\text { - Tout complexe à dominante (Pêcheux 1975) } \\
\text { - Détermine ce qui peut et doit être dit (articulé sous la } \\
\text { forme d'une harangue ... [etc.]) (Pêcheux 1971) } \\
\text { - Structuration de l'espace social par différenciation des } \\
\text { discours ; structure implicationnelle d'affectation d'effets de } \\
\text { sens aux registres (Achard 1995a : } 84,91 \text { ) }\end{array}$ \\
\hline Pratiques langagières & Registres discursifs \\
\hline $\begin{array}{l}\text { - Les pratiques langagières sont des pratiques sociales } \\
\text { - elles produisent des effets sur le social }\end{array}$ & $\begin{array}{l}\text { - Discours lié à une pratique sociale stable } \\
\text { - Pas d'existence en soi : lié à un point de vue } \\
\text { - (qui peut être celui de l'analyste mais aussi celui qui est } \\
\text { constitué par le système régulé des rapports sociaux ; la } \\
\text { pertinence sociale (= stable et partagée) du point de vue } \\
\text { que choisit l'analyste est condition d'une sociologie du } \\
\text { langage) } \\
\text { - [préstructuration de l'espace discursif en registres, 1985] } \\
\text { - Type d'actes. } \\
\text { - Circulation effective des discours entre des locuteurs } \\
\text { partageant une même place sociale. (Achard 1989) } \\
\text { - Matérialité locale qui s'appuie sur la stabilisation, par } \\
\text { répétition, d'une interpellation prenant une consistance } \\
\text { institutionnelle + ou - forte (Achard 1995a) } \\
\text { - Hypothèse }\end{array}$ \\
\hline \multirow[t]{2}{*}{ Non lié à un genre } & Lié à un genre \\
\hline & Genre discursif \\
\hline $\begin{array}{l}\text { - Ne suppose pas un rapport de motivation. } \\
\text { - Mais des régularités sont observées ; interprétables } \\
\text { seulement dans l'interdisciplinarité. }\end{array}$ & $\begin{array}{l}\text { - Suppose un rapport de motivation ou de convenance entre } \\
\text { pratique et formes } \\
\text { - Répartition inégale des formes motivée par un rapport } \\
\text { entre formes et fonctionnements sociaux (Achard 1995a) } \\
\text { - Ensemble de contraintes non arbitraire sur les marques } \\
\text { linguistiques (Achard 1995b) }\end{array}$ \\
\hline
\end{tabular}




\section{DES ECUEILS POUR LA MISE EN COMMUN}

Dans une présentation de l'analyse menée sur la passation de questionnaires lors de l'enquête «Analyse des comportements sexuels en France », les notions de registre et de pratiques langagières peuvent s'articuler, sans que, pour autant, on puisse régler la question du rapport à la formation langagière :

« Je prendrai ce matériel comme exemple d'administration orale de questionnaire considérée comme un registre discursif stabilisé. Pour cela, l'ensemble des transcriptions dont nous disposons ont été considérées comme un corpus lexicométrique unique (en mettant bout à bout toutes les transcriptions) où nous avons distingué deux parties : ce que dit l'enquêteur et ce que dit le sondé. » (Achard $1994: 84$, nous soulignons).

On peut émettre l'hypothèse que ce que dit l'enquêteur et ce que dit le sondé sont deux pratiques langagières différentes, en rapport l'une avec l'autre, sur un même terrain, le registre "passation de questionnaire », où du dialogisme joue, mettant l'enquêteur (en tant que place prise en charge) en position de dominant et le sondé en position de dominé. Qualifier le sondé de «dominé » est peut-être abusif, il n'en reste pas moins que lui n'est pas un professionnel tandis que l'enquêteur, même dilettante en est un, socialement parlant. On est, partant, conduit à se demander si registre et formation langagière ne sont pas en concurrence puisque cette formation est définie comme ensemble réglé de pratiques langagières et que, si notre hypothèse se tient, le 'réglage' de ces pratiques langagières ressortit d'un registre discursif.

Une autre façon de poser la question est de prendre appui sur l'hypothèse selon laquelle ces deux cadres sont deux points de vue différents sur un même objet empirique dégageant des objets de connaissance différents et éventuellement articulables.

Dans le cas des enquêtes, quelle qu'en soit la nature (sociologique, d'opinion ou autre), la passation de questionnaire est conçue comme un registre discursif chez Achard. Ainsi lit-on qu'une «caractéristique du registre 'passation d'enquête fermée' serait la dominance du discours de l'enquête, qui le distingue fortement du registre voisin de la passation des enquêtes par entretien » (Achard 1995b : 24, note 11). La question serait alors : qu'est-ce qui est mis en évidence par le biais d'une approche en termes de registre discursif, et qu'est-ce qui différencie cette approche de celle en termes de pratiques langagières? Au sein de ce type de discours, il nous semble que les sources apparaissent comme de bons critères pour poser l'hypothèse de pratiques différentes ; ainsi est-on amené à penser les productions de l'enquêteur et celles de l'enquêté comme deux pratiques langagières différentes, concernant un même thème, le questionnaire ou l'enquête en cours.

$\mathrm{Si}$ on trouve quelques échos des notions utilisées par un cadre dans l'autre, en revanche, des articulations entre ces appareils conceptuels ne nous semblent pas avoir été proposées par les auteurs. Il s'avère par ailleurs que l'importance attribuée à certaines notions n'est pas identique dans les deux cadres (celle de discours ou celle de langue par exemple).

\subsection{Des échos, des reprises et des déplacements}

\section{Formation Langagière chez Achard}

L'interprétation que propose Achard de formation langagière met bien en relation des pratiques langagières - des «codes » ou des «langues » en relation - tissant un réseau qu'on appelle formation et, puisqu'il la relie à la question du choix de code, à celle du répertoire linguistique et à celle de la répartition fonctionnelle, il reprend également la 
problématique des rapports de force. Néanmoins, il n'emploie jamais le terme, ni l'opposition entre pratiques dominantes et pratiques dominées. Peut-être faut-il voir là l'influence de la pensée de Foucault qui parle davantage en termes de pouvoir qu'en termes de domination (probablement pour laisser place à la prise en compte des pratiques marginales hors du champ des pratiques dominantes, en tant que telles).

\section{L'Analyse de Discours chez Ebel-Fiala}

«"Les mots changent de sens", dit-on. Cela veut dire aussi que les mots peuvent former entre eux des combinaisons nouvelles produisant des déplacements de signification. Durant les campagnes, ces déplacements s'accélèrent et se diversifient. De nouvelles tournures, de nouveaux mots surgissent dans les polémiques. Des expressions particulières deviennent des formules, énoncées sous des formes et dans des situations de langage les plus diverses. Ainsi, en est-il de «Ueberfremdung » et de «xénophobie » qui en tant que formules sont des enjeux centraux dans les débats, amplifiés et développés non seulement par les discours collectifs, mais encore au travers d'une multitude d'échanges verbaux particuliers. La signification de ces termes n'existe pas en dehors de ces paraphrases multiples et constamment déplacées où ils se trouvent utilisés, définis explicitement ou non. Ce ne sont donc pas seulement les conditions de production différentes qui déterminent des effets de sens différents, ce sont aussi les conditions de l'échange des discours, de leurs rencontres. » (Ebel-Fiala 1983 : 170, nous soulignons).

\subsection{Deux approches du discours et de l'énonciation}

Dans la perspective qui est la nôtre, de comparaison et d'articulation des cadres, la langue et le traitement qu'on en propose sont nodaux ; il faut ainsi pouvoir interroger la différence terminologique observable dans les notions des deux cadres et le choix d'adjectifs qui leur sont accolés : « langagier » et « discursif ». Il s'agit en outre de deux termes qui résultent de choix ; au moment où ils ont été opérés (fin 70-fin 80) ces choix terminologiques sont des démarcations et des prises de position théoriques.

Le terme «discours » est utilisé de manière très contrastée par les deux cadres. Il concerne toute production langagière (à la fois processus et produit) dans le cadre des Formations et pratiques langagières et renvoie à l'«usage du langage en situation pratique envisagé comme acte effectif et en relation avec l'ensemble des actes (langagiers ou non) dont il fait partie » chez Achard : «Le discours n'est pas la face langagière de l'acte, mais l'interdépendance de la face langagière et de la face pratique. » (1995: 84).

Les deux cadres se distinguent en outre par leur approche de l'énonciation - et plus largement de la langue. Chez Achard, prendre en compte l'énonciation consiste, outre s'intéresser à la construction de places sociales au sein de registres discursifs, à prendre acte des travaux théoriques de Benveniste et des développements en linguistique de l'énonciation chez Culioli. Chez J. Boutet, la filiation revendiquée à Culioli (Boutet, 1995) concerne les aspects dynamiques de l'ajustement intersubjectif entre les sujets parlants. Chez Ebel-Fiala, l'énonciation renvoie à Bakhtine-Volochinov (1929) et à la notion de parole vivante, située, qui se prolonge directement dans la notion de pratique langagière.

\section{Le traitement du pronom «nous »}

Pour comparer le traitement des formes linguistiques dans les deux démarches d'analyse, nous nous pencherons à présent sur le pronom «nous » dont les deux cadres traitent à un moment ou l'autre. Chez Ebel et Fiala, l'analyse de «nous » fait partie de 
l'observation des pratiques langagières de la presse suisse ayant eu cours durant la campagne pour les votations. Il semble que l'analyse ait l'argumentation pour visée :

«L'analyse des formes énonciatives du discours doit être menée d'un double point de vue ; d'une part on peut repérer des régularités formelles dans les combinaisons entre marques linguistiques. Mais en même temps ces régularités n'expriment pas simplement une combinatoire où les valeurs des divers éléments seraient purement abstraites, car les formes énonciatives sont toujours inscrites dans des pratiques langagières où les valeurs qui les opposent portent des rapports de forces et fondent divers effets qu'on assigne traditionnellement au langage. La signature d'un directeur politique, celle d'un rédacteur en chef, celle d'un lecteur particulier, mises en corrélation avec les formes énonciatives de leur discours ne forment pas des combinatoires propres qu'on pourrait interpréter ensuite comme différents faits de subjectivité dans le discours; ces signatures sont à la fois des traits de langage et des faits inscrits dans des rapports sociaux. [...] Si le nous du simple lecteur peut selon les cas renvoyer à un effet de subjectivité proprement dit, le nous d'une rédaction, inscrit dans une institution reconnue, circulant comme telle au sein d'une formation sociale, renverra nécessairement à des effets d'autorité, d'objectivité. On verra d'ailleurs plus loin comment, en publiant des lettres de lecteurs, c'est-à-dire en les mettant en circulation au sein de son propre discours, la rédaction d'un journal transforme nécessairement la valeur de telle ou telle forme énonciative produite par un simple lecteur dans son discours ». (Ebel et Fiala $1983: 254)$.

Ce sont les rapports de forces soutenus par les effets de sens d'autorité et/ou d'objectivité qui apparaissent ici comme objet de la description. Le poids de l'institution que constitue la rédaction d'un journal, mais plus généralement sans doute, la presse, intervient de façon cruciale dans la valeur argumentative des formes de l'appareil formel de l'énonciation. La dimension d'acte du langage conçu dans le cadre de pratiques langagières est ainsi réitérée, sur le plan des rapports de force :

«Rappelons que les pratiques langagières non seulement portent traces ou reflètent les rapports de forces existant dans une société, mais qu'elles instaurent des rapports de forces. Le fonctionnement du discours de la presse nous paraît à cet égard exemplaire : dans un journal la parole est distribuée à des locuteurs représentatifs à un titre ou à un autre (ces prises de parole reflètent les rapports de forces existants); en outre, par le type de cautions sociales qu'il recherche, le discours de la presse consolide les rapports de force existants ». (ibid. : 262, note 11)

On est donc en présence d'un processus de légitimation par appel à d'autres discours puisque les «cautions sociales » en question sont des experts (en économie la plupart du temps) ou des hommes politiques.

Ce n'est pas ce qui constitue l'objet de l'analyse des «nous » dans l'observation que propose Achard des dires tenus lors du Congrès des députés du peuple de l'Union soviétique de 1989 ( $c f$. Achard 1995b). Précisant ce qu'il entend par genre discursif, et resserrant son approche sur les marques formelles de la personne, Achard avance que

«... il est possible de s'appuyer sur la régularité de telles marques dans des corpus représentatifs du registre pour reconstituer une description vraisemblable des contraintes institutionnelles auxquelles les sujets qui prétendent à y occuper une place légitime sont soumis. La validité d'une telle démarche repose sur le double phénomène : de la valeur d'acte constructif du discours qui crée symboliquement les places que l'interprétation des marques linguistiques présuppose; de l'interprétation des sujets se trouvant en position indexicale d'être contraints d'agir dans le contexte des discours déjà émis et de ne pouvoir construire de nouveaux actes qu'en prenant en compte le déjà attesté dans le registre » (ibid. : 10). 
Après avoir montré des «'effets de genre' massifs » dans l'usage des pronoms personnels (je, nous et vous, au nominatif et à d'autres cas) lié au cas, c'est par la mise en relation de la distribution quantitative des formes et des différents groupes de « députés » ${ }^{11}$ que l'analyse débute. Prenant appui sur la caractéristique globale dégagée (lien entre le cas et la personne employée), la visée de l'analyse est différentielle : les différents groupes de députés ont-ils, localement donc — par rapport au « global» que représente l'ensemble des données - , un usage différent des marques de personne en fonction des cas? L'analyse d'Achard montre une différence entre les groupes, selon que les députés sont nommés ou élus; et selon qu'ils sont de Moscou, de la Fédération de Russie ou des autres Républiques de l'Union soviétique. Autrement dit, les textes sont rassemblés par l'analyste sur la base de l'hypothèse qu'il y a là une différence entre registres, c'est-à-dire entre groupes de députés, ce qui, sur le plan des marques de la personne renforcé par l'approche des cas, est confirmé. On observe une sorte de covariation entre registre et genre. Dans le même temps, le terme « registre »n'est utilisé qu'en référence au Congrès et non aux différents groupes; les différents groupes peuvent ici être envisagés comme ayant des pratiques langagières différentes, entrant en relation dialogique et constitutives du registre discursif du Congrès.

Adoptant ensuite une démarche «descendante» qui centre l'analyse sur l'interprétation de «u nas» («nous avons » ou «chez nous») en début de phrase, et non sans passer par une description abstraite des marques renvoyant à l'origine de l'énonciation (comme «nous » ou «nas» dans les deux langues, français et russe), Achard recense sept valeurs référentielles prises par cette forme dans son corpus. Ces valeurs permettent «de dégager une structure d'ensemble du champ pragmaticoénonciatif du registre discursif ». A partir de cette structure, qui joue le rôle d'un fond de carte, chaque occurrence est réinterprétée en fonction des rapports d'inclusion (spécifiée ou non) et d'exclusion. Chaque occurrence interprétée en fonction d'hypothèses externes générales permet de «construire un paradigme par recollement » (ibid. : 28), c'est-à-dire des places d'énonciation structurant le discours tenu dans ce registre (le Congrès des députés du peuple d'URSS, en 1989). C'est la conclusion à laquelle parvient Achard qui semble le mieux mettre en perspective sa démarche d'analyse sur les marques de la personne et leurs valeurs référentielles différentes non tant en fonction de qui parle que dans le cadre du débat qui a lieu à la Douma. Selon lui, l'appel au régional, effectué selon des modalités différentes examinées dans le cours de l'analyse, «permet de comprendre que l'éclatement de l'union ait pu être si rapide » (ibid. : 33).

C'est ainsi en terme de places que l'analyse se mène chaque fois, en tant qu'elles sont construites par les formes.

Toutefois, au sein de registres particuliers, Achard a essentiellement étudié le fonctionnement de l'appareil formel de l'énonciation et n'a considéré le lexique que du point de vue de son fonctionnement au sein de l'archive et de l'interdiscours. A la différence de certains travaux réalisés dans le cadre culiolien (entre autres, de Vogüe et Franckel 2002), on ne pourrait parler d'invariant de l'unité «grand » au même titre qu'on parle d'invariant de l'unité «nous ». L'analyse sémantique du lexique - et nous rejoignons ici les positions de Ebel-Fiala (1983) ou de J. Boutet (1982) - serait une projection de l'idéologique. Pour rendre compte du fonctionnement lexical, on aurait alors besoin d'une sémantique discursive (Achard, ms), ou d'une sémantique sociale (Boutet, 1982).

\footnotetext{
${ }^{11}$ Tous ne sont pas élus, certains étant nommés par les associations des Républiques.
} 


\section{EN CONCLUSION : TENTATIVE D'ARTICULATION DES DEUX CADRES}

\section{Le point de vue sur les données}

Nous émettons tout d'abord l'hypothèse qu'il est possible d'articuler ces deux cadres du point de vue de l'entrée sur les données et sur la constitution du corpus qu'ils supposent. Après avoir posé une hypothèse de registre discursif, c'est-à-dire une accumulation de textes dans un même voisinage socialement construit par une catégorisation stable (qui suppose un point de vue), qu'on peut repérer par une dénomination (telle que le discours de $\mathrm{X}$, ou l'école, ou la politique...), on peut décrire les pratiques langagières hétérogènes qui s'y font jour, et les processus de production de sens qui s'en dégagent, de par la circulation de formules. En ce sens, un registre discursif peut avoir pour fonction de cadrer les pratiques langagières, de les homogénéiser.

Nous estimons qu'une analyse en sociologie du langage ne peut s'en tenir à l'observation de ce processus et elle doit se donner les moyens de mettre en évidence les effets du langage dans le «comportement » des acteurs, dans l'organisation des rapports sociaux et dans le fonctionnement social. C'est probablement la catégorisation sociale des actes qui offre le biais le plus intéressant pour penser cette articulation.

Le problème que l'on peut rencontrer dans ce cas, et dans l'articulation des deux cadres théoriques, se situe entre

- l'affirmation de P. Achard, selon qui on peut associer un genre au registre discursif, c'est-à-dire des formes attestées dans ce lieu de discours qu'est le registre, formes qui entretiennent un certain rapport de motivation avec le type d'acte envisagé ; autrement dit, la notion de genre associé à un registre suppose une stabilité suffisante du sens (ou de la signification) pour qu'on puisse en dégager une sorte d'invariant qu'on observera dans son fonctionnement discursif et donc situé,

et

- l'affirmation de M. Ebel, P. Fiala ou de J. Boutet selon qui, par appui sur la théorie de la pluriaccentuation de M. Bakhtine, on ne peut partir de la signification des «mots », qui serait en langue (ce qu'ils récusent), mais qu'il faut envisager le sens en tant que processus. Si la notion de genre, qui prend appui sur une sorte d'invariant des mots (puisqu'il existe un rapport non-arbitraire entre les pratiques les registres discursifs - et l'énonciation), n'est pas utilisée dans le cadre théorique des pratiques langagières, c'est que ce dernier prend appui sur la théorie de Bakhtine-Volochinov (1929). Soulignant la contradiction du fonctionnement des formes linguistiques ${ }^{12}$, Ebel et Fiala précisent que c'est dans le prolongement de cette réflexion, qui cherche à montrer comment l'opposition/contradiction entre thème et signification travaille la langue, qu'ils utilisent les notions de pratique langagière et de formation langagière. (Ebel-Fiala 1983 : 150).

\section{Proposition de schématisation des relations}

Nous pouvons proposer le schéma suivant, qui montre les liens que ces différentes notions entretiennent selon nous. On y voit les décalages de niveau d'appréhension des phénomènes d'une part et le type de lien d'inclusion ou de subordination - formalisés par les cadres théoriques : en trait gras ou non formalisés par ces cadres mais que nous avons espéré montrer ici, en trait simple. L'affinité entre pratiques langagières et genre, nous semblant à développer a été notée en trait discontinu.

12 Unités à contenu invariable et, tout à la fois, unités idéologiques qui dépendent de la situation d'énonciation et du contexte discursif. «Contradiction » est sans doute à entendre au sens marxiste du terme, c'est-à-dire non pas un paradoxe, mais une dynamique entre des entités (signes ou classes sociales) opposées, dont le terme de dialectique rend probablement compte. 


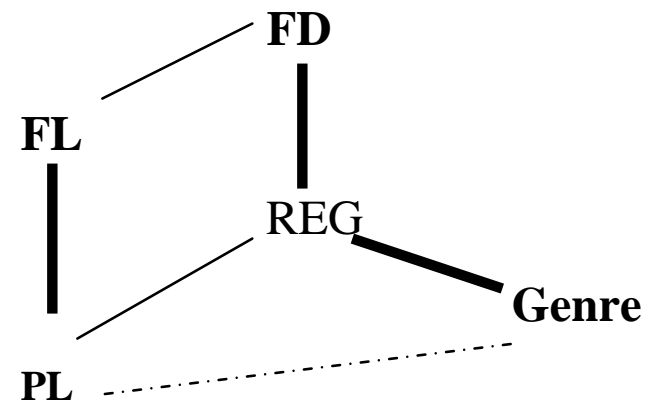

Une articulation au niveau de la matérialité langagière : variations et invariants

Nous émettons par ailleurs l'hypothèse qu'il est aussi possible d'articuler ces deux cadres au plan de la matérialité langagière, la langue. Nous dirons que la description d'une pratique langagière dans un registre discursif permet de mettre en évidence le poids institutionnel sur la circulation des discours ainsi que sur le processus de production de sens des textes. Ce qui est articulable au plan de la langue c'est alors l'invariant, supposé dans le cadre du rapport entre genre et registre discursif - une forme insistante n'étant pas réitérée «pour rien» ( $c f$. notamment Léglise 1999 et Cambon 2004) et la variation, supposée dans le cadre de l'observation des pratiques langagières ayant cours et s'opposant dans un registre discursif.

Stabilité et instabilité peuvent également être articulées du point de vue d'une description en sociologie du langage. Pour employer une métaphore picturale, on peut dire qu'en même temps qu'on offre un tableau, fixe, qui lisse les mouvements, mais, ce faisant, donne une vision de la stabilité (registres), on offre le film, qui restitue ces mouvements (les pratiques langagières), ces derniers ne mettant pas en cause, a priori, la stabilité de la pratique sociale observée (passation de questionnaire, Congrès politique, travail sur une chaîne, apprentissage en classe, formation professionnelle, campagnes politiques dans la presse générale ou partisane...). La mise en évidence de ces mouvements permet de montrer des changements qui ont cours dans les registres discursifs via les pratiques langagières.

\section{Références bibliographiques}

ACHARD Pierre (1984). «"Je jure..." (commentaire sur Ce que parler veut dire de Pierre Bourdieu)», Langage et Société $\mathrm{n}^{\circ} 29$, pp. 61-79.

- (1986). «Analyse de discours et sociologie du langage », Langage et Société n³7, pp.5-60.

- (1989). «Quelques propositions en sociologie du langage », in DRIGEARD Gabrielle, FIALA Pierre, TOURNIER Maurice (dir.) Courants sociolinguistiques (séminaire de lexicologie politique de l'Université Paris III, 1986-1987). Paris, InaLF-Klincksieck, pp. 39-51.

- (1993). La Sociologie du langage. Paris, PUF, Que sais-je?

- (1994). «Sociologie du langage et analyse d'enquête. De l'hypothèse de la rationalité des réponses », Sociétés contemporaines $\mathrm{n}^{\circ} 18-19$, pp. 67-100.

- (1995a). «Formation discursive, dialogisme et sociologie », Langages n¹17, pp. 82-95.

- (1995b). «Registre discursif et énonciation : induction sociologique à partir des marques de personne. Le Congrès des députés du peuple d'URSS en 1989 », Langage et Société n71, pp. 5- 
Cambon, Emmanuelle \& Léglise, Isabelle (2008). Pratiques langagières et registres discursifs : Interrogation de deux cadres en sociologie du langage. Langage et Société 124, 15-38

34.

- Fondements de sémantique discursive, ms.

AUTHIER-REVUZ Jacqueline (1995). Ces mots qui ne vont pas de soi. Boucles réflexives et noncö̈ncidences du dire, Paris, Larousse.

BAKHTINE-VOLOCHINOV (1975). Le Marxisme et la philosophie du langage. Paris, Minuit.

BAUTIER Élisabeth (1995). Pratiques langagières, pratiques sociales. De la sociolinguistique à la sociologie du langage, L'Harmattan.

BENVENISTE Émile (1966, 1974). Problèmes de linguistique générale. Tome 1 et Tome 2, Paris, Gallimard.

BOUTET Josiane (1982). «Matériaux pour une sémantique sociale », Modèles linguistiques Tome IV, fasc. 1, 7-37. Presses Universitaires de Lille.

- (1994). Construire le sens, Peter Lang.

- (1995). «Une linguistique de l'activité », in FRANCKEL J.-J. et ROBERT S., eds, Langues et langage. Problèmes et raisonnements en linguistique (Mélanges offerts à A. Culioli), Paris: Presses Universitaires de France.

- (2002). Pratiques langagières ; Formation langagière, in CHARAUDEAU P., MAINGUENEAU D., dir., Dictionnaire d'Analyse du Discours, Paris, Seuil.

BOUTET Josiane, FIALA Pierre, SIMONIN-GRUMBACH Jenny (1976). «Sociolinguistique ou sociologie du langage », Critique $\mathrm{n}^{\circ} 344,68-85$.

BOUTET Josiane, GARDIN Bernard et LACOSTE Michèle (1995). « Discours en situation de travail », Langages $n^{\circ} 117,12-31$, Paris : Larousse.

CAMBON Emmanuelle (2004). Personnification et personnalisation dans le discours politique du Front national. Approche discursive de la figure du représentant politique construite par les formes d'anthroponymes. Thèse de doctorat, Université Paris III.

COURTINE Jean-Jacques (1991). «Le discours introuvable : marxisme et linguistique (1965 - 1985), Histoires, Épistémologie, Langage, 13/II, 153-171.

COURTINE Jean-Jacques et MARANDIN Jean-Marie (1981). «Quel objet pour l'analyse du discours ? » dans Matérialité discursives, Lille, Presses Universitaires de Lille, 21-33.

DE VOGUE Sarah et FRANCKEL Jean-Jacques (2002). «Identité et variation de l'adjectif grand », Langue française 133, 28-41.

EBEL Marianne et FIALA Pierre (1983). Sous le consensus, la xénophobie. Paroles, arguments, contextes (1961-1981). Lausanne, Institut de science politique. Mémoires et documents 16.

FAYE Jean-Pierre (2004). Les Langages totalitaires (nouvelle édition), Paris, Hermann.

FOUCAULT Michel (1969). L’Archéologie du savoir. Paris, Gallimard.

- (1971). L'Ordre du discours. Paris, Gallimard.

Haroche Claudine, Henry Pierre, PecheuX Michel (1971). «La sémantique et la coupure saussurienne : langue, langage, discours », Langages $\mathrm{N}^{\circ} 24$.

LEGLISE Isabelle (1999). Contraintes de l'activité de travail et contraintes sémantiques sur l'apparition des unités et l'interprétation des situations, Thèse de doctorat, Paris, Université Paris 7 - Presses du Septentrion. 
Cambon, Emmanuelle \& Léglise, Isabelle (2008). Pratiques langagières et registres discursifs : Interrogation de deux cadres en sociologie du langage. Langage et Société 124, 15-38

MARCELLESI Jean-Baptiste, GARDIN Bernard (1974). Introduction à la sociolinguistique, LAROUSSE

PÊCHEUX Michel (1975). Les vérités de la palice. Linguistique, sémantique, philosophie, éd. F. Maspéro, Paris, $279 \mathrm{p}$.

- (1990). L'Inquiétude du discours. Textes réunis et présentés par Denise Maldidier, Paris, Éditions des Cendres.

ROBIN Régine (1973). Histoire et linguistique, Armand Colin, Paris, 307 p.

SLADE (1996). Sociologie du langage, analyse de discours et énonciation. Rapport scientifique à l'appui de la demande de création d'unité CNRS (Université Versailles - Saint-Quentin-en-Yvelines), 110 p. 